\title{
A First-Year Undergraduate Summer Assignment Designed to Introduce and Reinforce Good Work Concepts
}

\author{
Jennifer Adrienne Johnson, Mary Katherine Duncan, and Ashley Elizabeth Schoener
}

In fall 2011, the Bloomsburg University Good Work Initiative was launched. The initiative was tasked with developing, implementing, and assessing programs to encourage students to reflect upon the concept of good work (i.e., work that is excellent, ethical, and engaged). In this paper, we describe the 2014 summer assignment, which was developed using lessons learned over 3 years of good work programming at our university. The assignment was designed to first introduce students to good work concepts through a brief reading, then reinforce the concepts through two case analyses, and finally challenge students to apply their understanding of the concepts by writing an essay about their own professional role model of good work. Students demonstrated the ability to understand and effectively apply good work concepts and reported high levels of satisfaction with the program.

Good Work has been defined by three distinguished psychologists, Gardner, Csikszentmihalyi, and Damon (2001), as work that is excellent (high quality), ethical (socially responsible), and engaged (personally meaningful). The GoodWork Project is part of a larger initiative, The Good Project, housed at Harvard Graduate School of Education's Project Zero. Their website showcases good work initiatives from around the world and provides access to a variety of resources (http://thegoodproject.org).

In fall 2011, Joan Miller and authors Duncan and Johnson launched the Bloomsburg University Good Work Initiative (http://goodwork.bloomu.edu). Its mission is to advance undergraduates' understanding of what it means to do good work and develop programs and resources to advance their pursuit of good work at our university and beyond. To achieve this mission, our team has integrated good work concepts into curricular and extracurricular activities, provided opportunities for students to reflect upon their academic work, and strengthened the culture of good work on campus by highlighting role models of excellence, ethics, and

Jennifer Adrienne Johnson (jjohnson@bloomu.edu) is an Associate Professor of Psychology in the Department of Psychology at Bloomsburg University of Pennsylvania

Mary Katherine Duncan is a Joan and Fred Miller Distinguished Professor of Good Work in the Department of Psychology at Bloomsburg University of Pennsylvania

Ashley Elizabeth Schoener is in Department of Counseling Psychology at the University of North Dakota 


\section{engagement.}

Our programming has primarily focused on advancing first-year students' understanding of academic good work. In fall 2011 and fall 2012, we embedded good work programming into our university's ACT101/Educational Opportunities Program first-year seminars, and since 2012, we have developed annual summer assignments for all incoming first-year students. In our 2012 summer assignment, students watched a video and wrote two essays about a young man, Sam Eshaghoff, convicted of being paid to take the SAT for high school students (Johnson \& Duncan, 2013). In the essays, students noted that Eshaghoff probably thought he was demonstrating good work but also recognized that Eshaghoff did not actually achieve ethical work. We were pleased with the outcomes.

In our 2013 summer assignment, incoming first-year students reflected upon good work not only as students, but also as persons and citizens (Duncan \& Johnson, 2014). They wrote essays describing their 4-year goals as students, persons, and citizens; challenges they might face in each role; and plans for overcoming anticipated challenges. Students then visited our website (http:// goodwork.bloomu.edu) to learn about campus-based resources to help them pursue good work in each role. Student feedback regarding the assignment was positive; however, some students found it confusing to differentiate the three roles. Given the assignment's scale and complexity, we considered it more appropriate for students to complete later in their undergraduate careers.

In summer 2014, we created a new 3-part summer assignment informed by previous Good Work programming at our university and supported by pedagogical literature suggesting the utility of providing multiple opportunities for students to learn about a concept. In the assignment, students were first introduced to good work concepts through a brief reading. Then, concepts were reinforced through two case analyses. Finally, students applied their understanding by writing essays about their professional role models of good work.

\section{Method}

Approximately 2,000 students ( $~ 80 \%$ of incoming first-year students) completed the 2014 summer assignment. Of these, 99\% were traditional-aged (1719 years old), 61\% were female, and 23\% reported being first-generation college students. As mandated by our university's Institutional Review Board, students were informed that their assignment responses would be combined with other students' responses and used for research purposes.

The assignment included three parts that students completed on our university's online course-management system prior to the fall 2014 semester. Part 1 introduced the concept of good work through a 2-page reading written by Johnson and Duncan (available at http://goodwork.bloomu.edu). The reading described the three Es of good work and common misconceptions about good work identified through our previous programming. We described excellence as built upon hard work and determination, but achieved through the demonstration of high-quality work. We described ethics as socially responsible behavior (putting the good of others above personal gains) and related ethics to our university's 
academic integrity policy. Finally, we described engagement as finding personal meaning in one's work and shared stories of personally fulfilling work by two students at our university. After the reading, students' understanding of good work was assessed through a multiple choice quiz.

In Part 2, students reinforced their understanding of good work by analyzing two case studies. The first case, Sam Eshaghoff, was the same used in the 2012 summer assignment because we thought it was effective. After watching the video, students answered four yes/no questions (i.e., Did Eshaghoff demonstrate excellent work? ethical work? engaged work? good work?) and explained their reasoning in one to two sentences. Authors Johnson and Duncan created the second case about a hypothetical college student, Alex, who completed an exam and, at the request of a close friend, informed the friend about some of the test questions. Students then answered one yes/no question (i.e., Did Alex demonstrate good work?) and explained their reasoning in three to five sentences.

In Part 3, students applied their understanding of good work by writing a 300500 word essay describing how their professional role model demonstrated each $E$. The role model could be someone they knew personally, someone from history, or a contemporary figure.

After completing the assignment, students indicated which part they found most valuable and reported their overall satisfaction with the assignment on a scale of 1 (low) to 10 (high). Students were also directed to our website (http:// goodwork.bloomu.edu) to learn more about campus-based resources to help them in their pursuit of good work.

\section{Results and Discussion}

Students' performance on the Part 1 quiz was very good (98\% correct overall). Although students were given immediate feedback and were able to change their responses until achieving the correct answer, their impressive performance suggests they were either correct at the start or were attending to the feedback and changing their responses accordingly. Interestingly, when students were asked which part of the assignment was most valuable, $48 \%$ chose Part 1 . This finding has encouraged us to use the reading to introduce future students to good work concepts.

In Part 2, most students (73\%) correctly identified Sam Eshaghoff's work as excellent because he earned very high scores on the SAT. Most students (89\%) also correctly identified Eshaghoff's work as not ethical because he was not putting the good of all above his own financial gains. Regarding engagement, it was unclear from the video whether Eshaghoff found his work personally meaningful (engaged) or if he was just interested in the financial gain (not engaged). Accordingly, we did not judge either response as correct. Overall, students skillfully used concepts learned in Part 1 to answer questions about Eshaghoff's work. On the other hand, $40 \%$ incorrectly identified Eshaghoff as a good worker despite reading in Part 1 that good work requires all three Es. To explore students' misunderstanding, we examined a random sample of 50 essays from the $40 \%$ of students who identified Eshaghoff as a good worker. Most students in the random 
sample (82\%) correctly recognized that Eshaghoff did not act ethically; however, many of these students thought Eshaghoff's unethical behavior was justified because he conducted a successful business. In future programming, we will emphasize that all three Es must be present for good work, and the outcome of one's work does not justify the means by which it was accomplished.

Students demonstrated a better understanding of good work in the second case about Alex. Most students (89\%) correctly reported that Alex did not demonstrate good work because sharing exam questions is against academic integrity standards (not ethical). We examined a random sample of 50 essays from the $11 \%$ of students who misidentified Alex as a good worker. Students in the random sample commonly misinterpreted Alex's loyalty to the friend as ethical behavior. Nonetheless, we were pleased to find that most students correctly recognized Alex's work as unethical because it compromised the integrity of the academic community.

In Part 3, students' choices of professional role models were predominantly family members $(60 \%)$, historical figures (15\%), and teachers or coaches $(15 \%)$. We chose a random sample of 100 essays and analyzed students' explanations of how their role models demonstrated each $E$ of good work. Most students (73\%) correctly described excellent work as high-quality work. Most students (63\%) also correctly described ethical work as putting the good of others above personal gains; however, some students incorrectly described ethical work as simply fulfilling basic professional responsibilities. We will address this misconception in future programming. Finally, most students (79\%) correctly described engaged work as finding personal meaning in one's work.

Most students (80\%) selected an overall satisfaction rating of 8 or higher for the assignment, which was encouraging, given that it was a mandatory assignment completed over the summer. Overall, our findings suggest that the assignment was an effective way to educate incoming first-year students about good work and encourage students to reflect on their commitment to academic excellence, ethics, and engagement as they transition to college. We hope this and future programming will continue to strengthen the culture of good work at our university.

\section{References}

Duncan, M. K., \& Johnson, J. A. (2014). Bloomsburg University Good Work Initiative: Marking undergraduates' path to success as students, persons, and citizens. Journal of College Orientation and Transition, 21(2), 49-53.

Gardner, H., Csikszentmihalyi, M., \& Damon, W. (2001). Good Work: When excellence and ethics meet. New York, NY: Basic Books.

Johnson, J. A., \& Duncan, M. K. (2013). Using the GoodWork ${ }^{\mathrm{TM}}$ model to introduce first-year students to the expectations of academia. Journal of College Orientation and Transition, 21(1), 88-92. 